\title{
Properties of Astaxanthin/ $\mathrm{Ca}^{2+}$ Complex Formation in the Deceleration of Cis/Trans Isomerization
}

2007

Vol. 9, No. 16 2985-2988

\author{
Chien-Sheng Chen, ${ }^{\dagger,}$ Shih-Hsiung Wu, ${ }^{*}, \neq$ Ying-Ying Wu,,${ }^{\S}$ Jim-Min Fang, ${ }^{\dagger}$ and \\ Tzu-Hua Wu ${ }^{\star}, \S$
}

Department of Chemistry, National Taiwan University, Taipei 106, Taiwan, Institute of Biological Chemistry, Academia Sinica, Taipei 115, Taiwan, and College of Pharmacy, Taipei Medical University, Taipei 110, Taiwan

shwu@gate.sinica.edu.tw; thwu@tmu.edu.tw

Received April 24, 2007

\section{ABSTRACT}

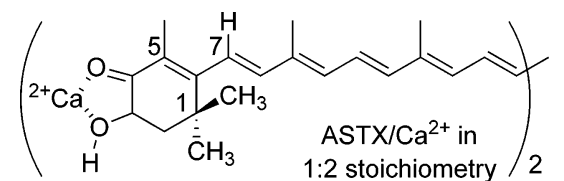

Deceleration of the regioselective cis/trans isomerization of all-trans-astaxanthin (ASTX) in the presence of Ca ${ }^{2+}$ was shown by HPLC analysis. The NMR and ITC analyses provided evidence for complexation of ASTX with $\mathrm{Ca}^{2+}$ in 1:2 stoichiometry via chelation at both carbonyl and hydroxyl groups. The rotation across torsion $\omega 6(\mathrm{C} 5-\mathrm{C} 6-\mathrm{C} 7-\mathrm{C} 8)$ upon complexation is consistent with the NOE between $7-\mathrm{H}$ and $5-\mathrm{CH}_{3}$. This study supports the inhibitory effect of ASTX on calcium-induced turbidity of lens crystallins.

Astaxanthin (3,3'-dihydroxy- $\beta, \beta^{\prime}$-carotene-4,4'-dione, ASTX, Scheme 1) is one of the most common xanthophylls found in the red pigment of crustacean shells (e.g., of crab and shrimp), salmon, and asteroideans. ${ }^{1}$ ASTX has several biological activities, including immuno-modulating activity, ${ }^{2}$ hepatoprotection, ${ }^{3}$ and protection of islet $\beta$-cells, ${ }^{4}$ and may be used in disease prevention, e.g., antihypertension, and in protection against ischemia-induced memory impairment. ${ }^{5}$ ASTX appears to be a more powerful antioxidant than other carotenoids and vitamin $\mathrm{E}$ in conferring numerous health benefits. ${ }^{6}$

\footnotetext{
National Taiwan University.

$\doteqdot$ Academia Sinica.

$\S$ Taipei Medical University.

(1) (a) Miki, W.; Yamaguchi, K.; Konosu, S. Comp. Biochem. Physiol., Part B: Biochem. Mol. Biol. 1982, 71, 7-11. (b) Ohgami, K.; Shiratori, K.; Kotake, S.; Nishida, T.; Mizuki, N.; Yazawa, K.; Ohno, S. Vestn. Ophthalmol. Vis. Sci. 2003, 44, 2694-2701.

(2) (a) Chen, C. J.; Tsai, C. C.; Hsieh, J. F.; Chien, C. M.; Wu, T. H.; Chen, S. T. Comb. Chem. High Throughput Screening 2006, 9, 777-784. (b) Chew, B. P.; Park, J. S. J. Nutr. 2004, 134, 257S-261S.

(3) (a) Kang, J. O.; Kim, S. J.; Kim, H. Methods Find Exp. Clin. Pharmacol. 2001, 23, 79-84. (b) Kozuki, Y.; Miura, Y.; Yagasaki, K. Cancer Lett. 2000, 151, 111-115.

(4) Uchiyama, K.; Naito, Y.; Hasegawa, G.; Nakamura, N.; Takahashi, J.; Yoshikawa, T. Redox Rep. 2002, 7, 290-293.

(5) Hussein, G.; Sankawa, U.; Goto, H.; Matsumoto, K.; Watanabe, H. J. Nat. Prod. 2006, 69, 443-449.
}

In nature, carotenoids exist predominately in the all-trans configuration. The long chain of conjugated carbon-carbon double bonds within carotenoids is susceptible to light,

Scheme 1. Structure of trans-Astaxanthin, 9-cis-Astaxanthin, and 13-cis-Astaxanthin

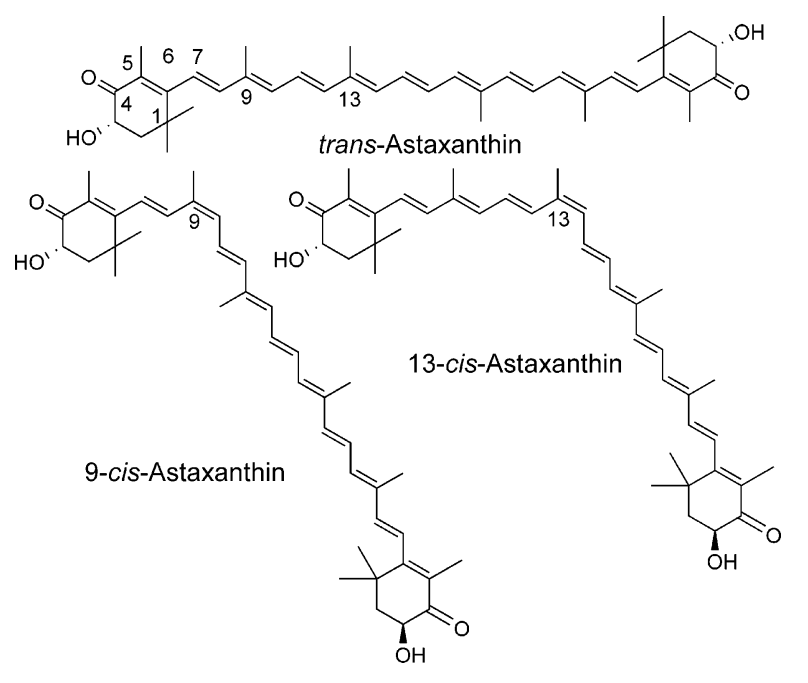


oxygen, heat, and acid degradation. The cis/trans configuration of the geometrical isomers greatly influences the overall molecular shape. The all-trans isomer of ASTX predominates in nature, but the 9-cis, 13-cis, and 15-cis isomers are also encountered. The occurrence of the isomerization of the all-trans isomer to the cis-isomers during the extraction of pigments, saponification of the trans-ASTX ester, purification of trans-ASTX, and preparation of transASTX standard solutions has been studied by using various organic solvents. ${ }^{7}$ The facilitation of the isomerization of trans-ASTX to the cis isomers induced by copper ions in ethanol has also been reported; however, the UV spectroscopy and HPLC analysis data are limited, and no detailed information on the structure of ASTX complexes has been provided. ${ }^{8}$

We previously showed that ASTX inhibits calciuminduced lens turbidity and that the ASTX/ $\mathrm{Ca}^{2+}$ interaction may be responsible for part of this protective effect. ${ }^{9}$ In fact, calcium and calcium-dependent enzymes play specific roles in the development of human diseases and symptoms, including cataracts, impaired cognition, and insulin resistance. ${ }^{10}$ Therefore, we aimed to investigate the chelation of calcium by ASTX and the chemical interactions between calcium and ASTX in order to provide meaningful interpretation for the in vivo and in vitro activities of ASTX.

Effect of $\mathrm{Ca}^{2+}$ on Isomerization. The stoichiometry of ASTX/Ca ${ }^{2+}$ in acetonitrile (ACN)/chloroform $(1: 1, \mathrm{v} / \mathrm{v})$ and $\mathrm{ACN} / \mathrm{CH}_{2} \mathrm{Cl}_{2}(1: 1, \mathrm{v} / \mathrm{v})$ was analyzed by NMR and isothermal titration calorimetry (ITC), respectively. ACN was used since it increased the solubility of $\mathrm{Ca}\left(\mathrm{ClO}_{4}\right)_{2}$. The formation of the ASTX/Ca ${ }^{2+}$ complex within 3 min of adding calcium ions was examined by using UV/vis spectroscopy. The absorbance increased slightly as the molar ratio of calcium ions increased, but the increase was not linear. The observed slight red-shift of the $\lambda_{\max }$ from 480 to $484 \mathrm{~nm}$ (see the Supporting Information) might result from a slightly better conjugation system in the polyene-backbone of this ketocarotene owing to the ASTX/Ca ${ }^{2+}$ complex formation. The results are consistent with (i) the lack of an apparent charge transfer because of the binding of two calcium ions at both ends of ASTX and (ii) the retention of the all-trans configuration of ASTX during the titration. In comparison, incubation of trans-ASTX with $\mathrm{Cu}^{2+}$ shows a large blueshift from 480 to $373 \mathrm{~nm}$ with decreased intensity in ethanol, ${ }^{8}$ presumably due to the accelerated isomerization to 9-cisand 13-cis-ASTX having the polyene chromophore twisted. The cis/trans isomerization of trans-ASTX to 9-cis or 13cis forms has been reported with first-order and reversible reaction kinetics in both $\mathrm{CH}_{2} \mathrm{Cl}_{2}$ and $\mathrm{ACN}$ solutions, ${ }^{11}$ and such an isomerization can be accelerated by thermal incubation..$^{7}$ Since we incubated samples under $37^{\circ} \mathrm{C}$ in earlier

(6) Guerin, M.; Huntley, M. E.; Olaizola, M. Trends Biotechnol. 2003, 21, 210-216.

(7) Yuan, J. P.; Chen, F. J. Agric. Food Chem. 1999, 47, 3656-3660.

(8) Zhao, L.; Chen, F.; Zhao, G.; Wang, Z.; Liao, X.; Hu, X. J. Agric. Food Chem. 2005, 53, 9620-9623.

(9) Wu, T. H.; Liao, J. H.; Hou, W. C.; Huang, F. Y.; Maher, T. J.; Hu, C. C. J. Agric. Food Chem. 2006, 54, 2418-2423.

(10) Carragher, N. O. Curr. Pharm. Des. 2006, 12, 615-638.

(11) Yuan, J. P.; Chen, F. Food Chem. 2001, 73, 131-137. lens studies, we also incubated the samples at $37^{\circ} \mathrm{C}$ in the current study. The trans-ASTX isomerization upon chelation with calcium ions was then analyzed by HPLC. Isomerization decelerated when trans-ASTX was incubated with calcium ions at an ASTX/Ca ${ }^{2+}$ molar ratio of 1:2 (Figure 1); similar
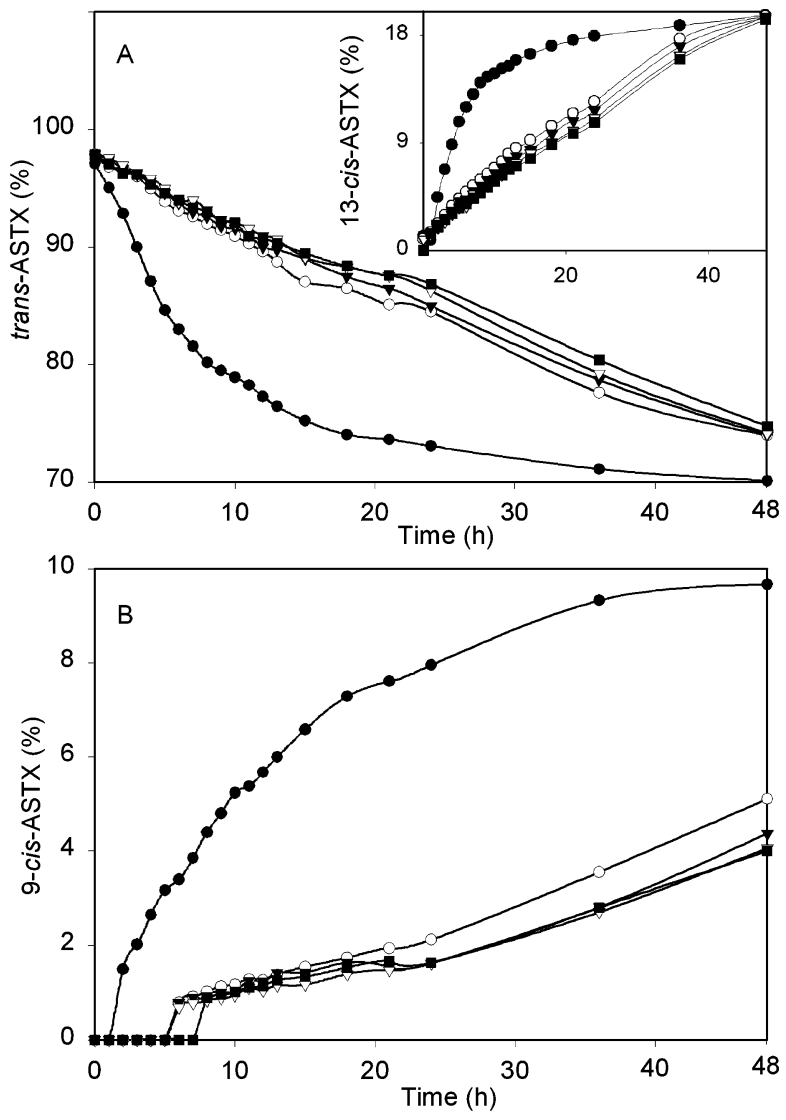

Figure 1. The cis/trans isomerization of ASTX in the presence of calcium at $37{ }^{\circ} \mathrm{C}$. The relative amount of (A) trans-astaxanthin, 13-cis-astaxanthin (insert), and (B) 9-cis-astaxanthin in $\mathrm{CH}_{2} \mathrm{Cl}_{2}$ / $\operatorname{ACN}(1 / 1, v / v)$ in the presence or absence of $\mathrm{Ca}\left(\mathrm{ClO}_{4}\right)_{2}$ are shown: (@) ASTX; (O) ASTX/Ca ${ }^{2+}(1: 2, \mathrm{~mol} / \mathrm{mol})$; ( $)$ ASTX/ $\mathrm{Ca}^{2+}(1: 4, \mathrm{~mol} / \mathrm{mol}) ;(\nabla) \operatorname{ASTX} / \mathrm{Ca}^{2+}(1: 8, \mathrm{~mol} / \mathrm{mol}) ;$ and $(\boldsymbol{\square})$ ASTX/Ca ${ }^{2+}(1: 16, \mathrm{~mol} / \mathrm{mol})$.

results were obtained with a molar ratio of 1:4. These results differed from the previously reported acceleration of cis/ trans isomerization of ASTX induced by $\mathrm{NaCl}^{7}$ and by copper ions, ${ }^{8}$ both of which were for different reaction times and at different temperatures. However, the observed regioselectivity, e.g., production of predominantly 13-cis-ASTX $(\sim 18 \%)$ after thermal incubation and a decrease $(\sim 6 \%)$ in 9-cis-ASTX formation in the presence of calcium after 48 $\mathrm{h}$, was similar to the results of other studies. ${ }^{7,8}$

Interaction of ASTX with $\mathbf{C a}^{2+}$. The binding properties of ASTX (2 mM) with calcium ions in ACN/chloroform (1: $1, \mathrm{v} / \mathrm{v})$ were evaluated by NMR. Addition of $\mathrm{Ca}^{2+}$ to ASTX at various molar ratios (ASTX/Ca ${ }^{2+} 1: 1$ to $1: 128$ ) caused downfield shifts in all proton signals of cyclohexenone moiety (Table 1). The most significant proton chemical shifts 
Table 1. ${ }^{1} \mathrm{H}$ and ${ }^{13} \mathrm{C}$ NMR Chemical Shifts (ppm) of ASTX and ASTX/Ca ${ }^{2+}$ Complex in ACN/Chloroform ${ }^{\mathrm{a}}$

\begin{tabular}{lccccccr}
\hline proton & $\delta_{\text {ASTX }}^{\mathrm{H}}$ & $\delta_{\text {complex }}^{\mathrm{H}}$ & $\Delta \delta_{\text {shift }}^{\mathrm{H}}{ }^{b}$ & carbon & $\delta_{\text {ASTX }}^{\mathrm{C}}$ & $\delta_{\text {complex }}^{\mathrm{C}}$ & $\Delta \delta_{\text {shift }}^{\mathrm{C}}{ }^{b}$ \\
\hline $\mathrm{CH}_{3}{ }^{\text {pro-S }}$ & 1.32 & 1.35 & 0.02 & $\mathrm{CH}_{3}{ }^{\text {pro }-S}$ & 24.75 & 24.46 & -0.29 \\
$\mathrm{CH}_{3}{ }^{\text {pro }}-R$ & 1.20 & 1.25 & 0.05 & $\mathrm{CH}_{3}{ }^{\text {pro }}-R$ & 29.39 & 29.27 & -0.12 \\
$\mathrm{H} 2$ & 2.14 & 2.16 & 0.02 & $\mathrm{C} 1$ & 35.69 & 36.41 & 0.72 \\
$\mathrm{H} 2^{\prime}$ & 1.76 & 1.91 & 0.15 & $\mathrm{C} 2$ & 44.40 & 44.16 & -0.24 \\
$\mathrm{H} 3$ & 4.29 & 4.73 & 0.44 & $\mathrm{C} 3$ & 68.16 & 70.42 & 2.26 \\
$3-\mathrm{OH}$ & 3.61 & 5.29 & 1.68 & $\mathrm{C} 4$ & 199.32 & 201.92 & 2.60 \\
$\mathrm{H} 7$ & 6.46 & 6.56 & 0.10 & $\mathrm{C} 5$ & 125.85 & 125.79 & -0.06 \\
$\mathrm{H} 8$ & 6.27 & 6.29 & 0.02 & $\mathrm{C} 6$ & 160.96 & 164.74 & 3.78 \\
$8-\mathrm{CH}{ }_{3}$ & 1.89 & 1.91 & 0.02 & $\mathrm{C} 7$ & 140.95 & 142.42 & 1.47 \\
$\mathrm{H} 10$ & 6.45 & 6.49 & 0.04 & $\mathrm{C} 8$ & 122.78 & 122.17 & -0.61 \\
$\mathrm{H} 12$ & 6.33 & 6.38 & 0.05 & $\mathrm{C} 9$ & 133.97 & 133.92 & -0.05
\end{tabular}

${ }^{a}$ ASTX, astaxanthin $(1 \mathrm{mM})$ in $\mathrm{ACN}-d_{3} /$ chloroform- $d_{1}(1: 1)$. The complexation of ASTX/Ca ${ }^{2+}$, ASTX $(1 \mathrm{mM})$ and $\mathrm{Ca}\left(\mathrm{ClO}_{4}\right)_{2},(1: 32, \mathrm{~mol} /$ mol). ${ }^{b} \Delta \delta_{\text {shift }}^{\mathrm{H}}=\delta_{\mathrm{ASTX} / \mathrm{Ca}}^{\mathrm{H}}-\delta_{\mathrm{ASTX}}^{\mathrm{H}} \cdot \Delta \delta_{\text {shift }}^{\mathrm{C}}=\delta_{\mathrm{ASTX} / \mathrm{Ca}}^{\mathrm{C}}-\delta_{\mathrm{ASTX}}^{\mathrm{C}} \cdot$

were the hydroxyl (3-OH, $\left.\Delta \delta_{\text {shift }}=1.68 \mathrm{ppm}\right)$ and $\alpha$ proton of the ketone group $\left(\mathrm{H} 3, \Delta \delta_{\text {shift }}=0.44 \mathrm{ppm}\right)$. The 2-D heteronuclear ${ }^{1} \mathrm{H}-{ }^{13} \mathrm{C}$ HMQC $\left({ }^{1} J\right)$ and HMBC $\left({ }^{2} J\right.$ and $\left.{ }^{3} J\right)$ also showed downfield shifts in the carbon nuclei, including $\mathrm{C} 3\left(\Delta \delta_{\text {shift }}=2.26 \mathrm{ppm}\right)$ and $\mathrm{C} 4\left(\Delta \delta_{\text {shift }}=2.60 \mathrm{ppm}\right)$. These results strongly indicate that the $\alpha$-hydroxylketone utilizes the lone pairs at the 3-oxygen and 4-carbonyl groups as the functional site involved in calcium chelation.

A clockwise rotation of the polyene chain across bond C6-C7 was suggested by the ROESY spectra of ASTX with and without calcium. The observed additional NOE of the ASTX/Ca ${ }^{2+}$ (1:32) complex, 7-H/5- $\mathrm{CH}_{3}$ (Figure 2B), was used in the molecular simulation with a distance restraint set at medium range (3.0-4.0 $\mathrm{\AA})$. The results led to a model with a torsion $\omega 6(\mathrm{C} 5-\mathrm{C} 6-\mathrm{C} 7-\mathrm{C} 8)$ of $-117.0^{\circ}$. This significant twist also reflects the nuclear downfield shift of C6 $\left(\Delta \delta_{\text {shift }}=3.78 \mathrm{ppm}\right), \mathrm{C} 7\left(\Delta \delta_{\text {shift }}=1.47 \mathrm{ppm}\right)$, and $\mathrm{H} 7$ $\left(\Delta \delta_{\text {shift }}=0.10 \mathrm{ppm}\right)$. This structure contrasts the previously reported X-ray analysis of uncomplexed ASTX with $\omega 6$ torsion of $59.3^{\circ}$ (Figure 2A)..$^{12}$ Our chelation model of ASTX $\left(\mathrm{Ca}^{2+}\right)_{2}$ complex indicated a better $\pi$-electron conjugation system of the cyclohexenone moiety to polyene, ${ }^{13}$ and the metal ion induced conformational regidification ${ }^{14}$ thus provided a reasonable explanation for the increase in absorbance. Because the 9-alkenyl group is closer to the calcium-chelation site, it would be more easily stabilized in the presence of calcium ions. This model of calciumchelated ASTX could also explain why the isomerization of trans-ASTX gave more 13-cis isomer than 9-cis isomer in the presence of calcium ions.

Thermodynamics of the ASTX/Ca ${ }^{2+}$ Complex. The measured absorbance of a chromophore can usually be used to characterize the binding properties of a ligand/metal

(12) (a) Moss, G. P. Pure Appl. Chem. 1979, 51, 507-514. (b) http:// xray.bmc.uu.se/hicup/.

(13) (a) Weesie, R. J.; Jansen, F. J.; Merlin, J. C.; Lugtenburg, J.; Britton, G.; de Groot, H. J. Biochemistry 1997, 36, 7288-7296. (b) Fiedor, J.; Fiedor, L.; Kammhuber, N.; Scherz, A.; Scheer, H. Photochem. Photobiol. 2002, 76, 145-152. (c) Gao, Y.; Focsan, A. L.; Li, Y. Y.; Kispert, L. D. J. Phys. Chem. A 2006, 110, 10091-10097. (d) Samokyszyn, V. M.; Freyaldenhoven, M. A.; Chang, H. C.; Freeman, J. P.; Compadre, R. L. Chem. Res. Toxicol. 1997, 10, 795-801.

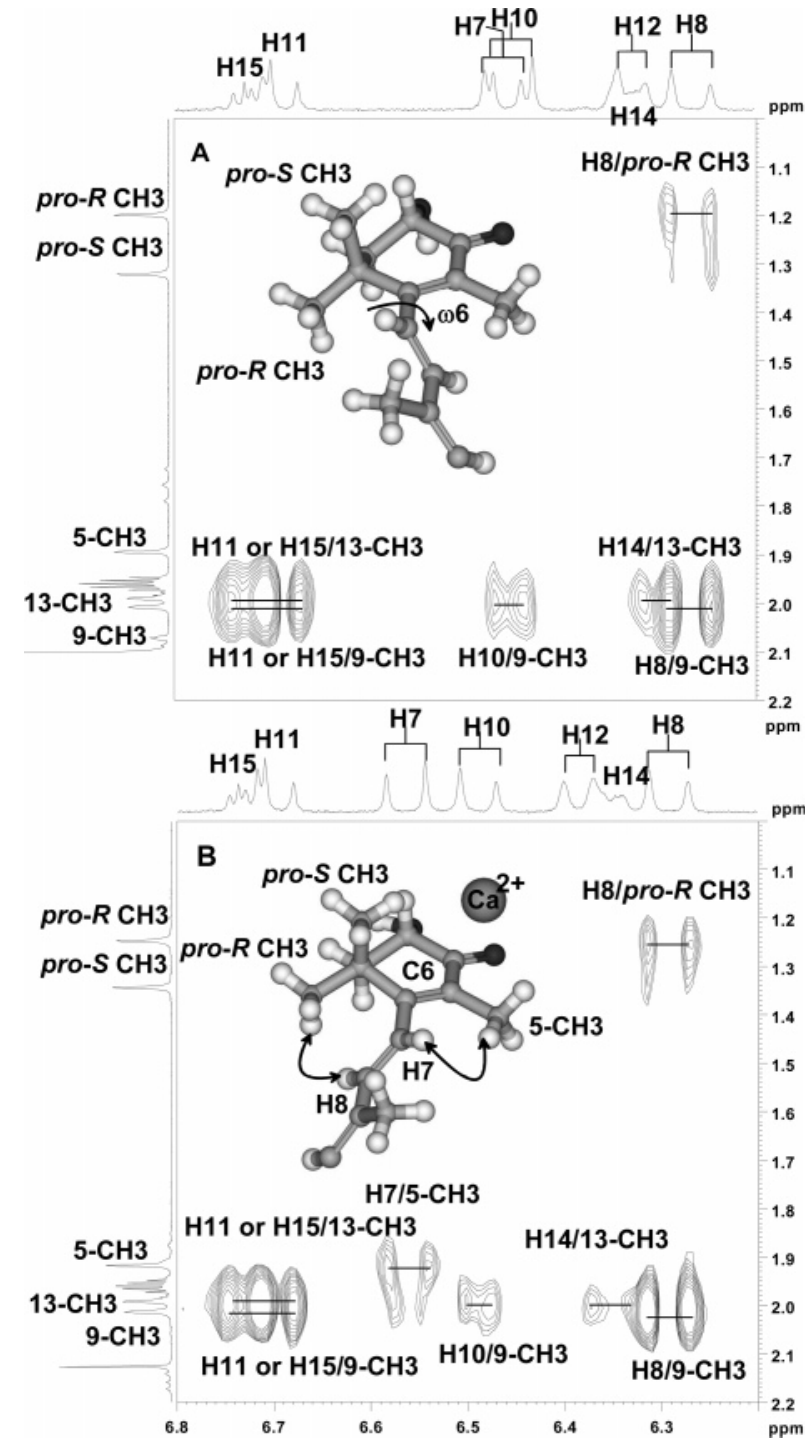

Figure 2. Partial ROESY spectra of ASTX (2 mM) alone (A) and with 32 molar equiv of calcium ions (B) with a mixing time of $300 \mathrm{~ms}$. The structural model in part A shows the partial 3-D structure of ASTX from the X-ray databank that matches the data obtained by ROESY. The structural model of ASTX/Ca ${ }^{2+}$ in part $\mathrm{B}$ is based on molecular simulation and indicates a clockwise rotation of the polyene chain across bond $\mathrm{C} 6-\mathrm{C} 7$ when compared to the native ASTX.

complex, ${ }^{14}$ but it is difficult to obtain this characterization without an isosbestic point in the UV/vis spectra. We used a Job plot to study the various concentrations of ligand/metal pairs following a design similar to that used in NMR analyses..$^{15}$ The results obtained by monitoring the downfield shifts of H3 of ASTX suggested that ASTX complexes two calcium ions (Table 1). By comparison with the literature, ${ }^{16}$

(14) (a) Liao, J. H.; Chen, C. T.; Chou, H. C.; Cheng, C. C.; Chou, P. T.; Fang, J. M.; Slanina, Z.; Chow, T. J. Org. Lett. 2002, 4, 3107-3110. (b) Kuo, L. J.; Liao, J. H.; Chen, C. T.; Huang, C. H.; Chen, C. S.; Fang, J. M. Org. Lett. 2003, 5, 1821-1824.

(15) Braun, S.; Kalinowski, H.-O.; Berger, S. 100 and More Basic NMR Experiments; VCH Publishers: New York, 1996. 
Time (min)

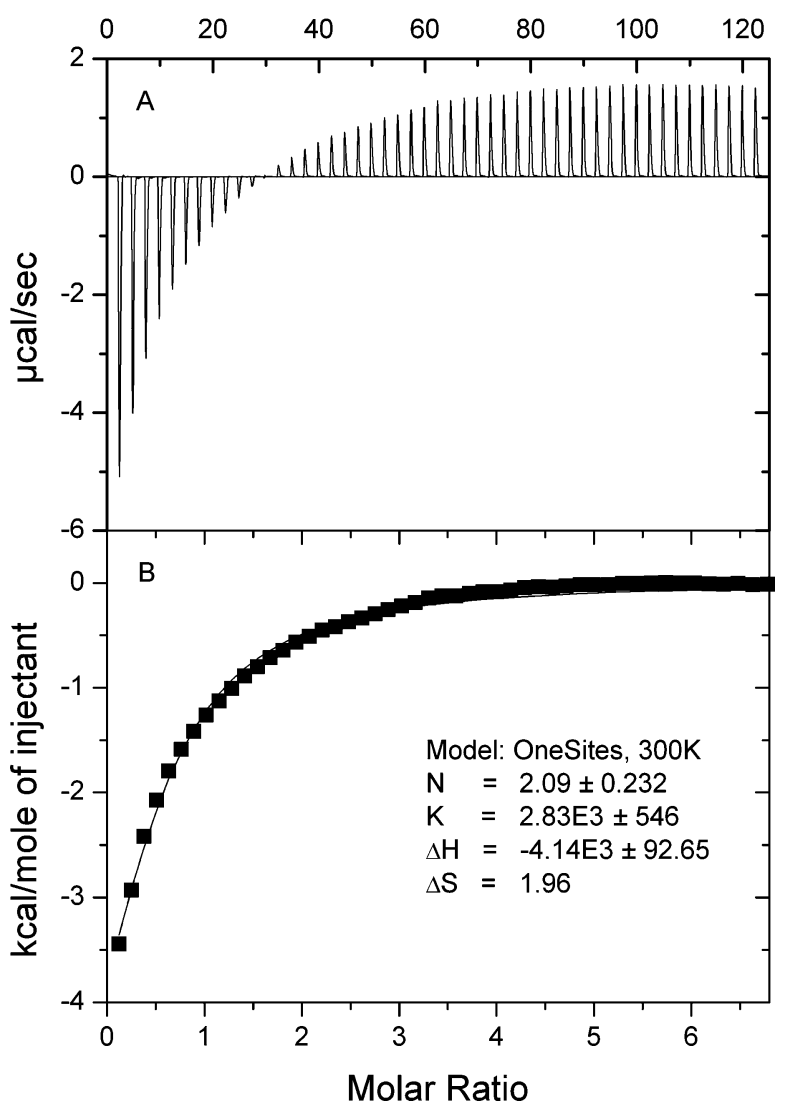

Figure 3. Isothermal titration calorimetry of the complex formation of ASTX $(0.25 \mathrm{mM})$ and calcium ions $(7.5 \mathrm{mM})$ at $300 \mathrm{~K}$. (A) Raw data obtained from 48 automatic injections (6 $\mu \mathrm{L}$ each) of $\mathrm{Ca}^{2+}$ and (B) the integrated fitting curve showing the experimental points with the value of the parameter, $n=2$, by using a one-site binding fitting function.

our ITC results (Figure 3) indicated that the association constant ( $K_{\text {ass }}$ ) of the ASTX/ $\mathrm{Ca}^{2+}$ complex prepared in $\mathrm{CH}_{2}-$ $\mathrm{Cl}_{2} / \mathrm{ACN}(1: 1)$ was $2830 \pm 546\left(\mathrm{M}^{-2}\right)$ at $300 \mathrm{~K}$ and the molar ratio was 1:2. In addition, the results of ITC showed not only a favorable enthapy change $(\Delta H=-4140 \pm 95.65$ $\mathrm{cal} / \mathrm{mol})$ but also an increase in entropy $(\Delta S=1.96 \mathrm{cal} /$

(16) (a) Haj-Zaroubi, M.; Mitzel, N. W.; Schmidtchen, F. P. Angew. Chem., Int. Ed. Engl. 2002, 41, 104-107. (b) Linton, B. R.; Goodman, M. S.; Fan, E.; van Arman, S. A.; Hamilton, A. D. J. Org. Chem. 2001, 66, 7313-7319. (c) Davis, A. P.; Wareham, R. S. Angew. Chem., Int. Ed. Engl. 1999, 38, 2978-2996. $(\mathrm{mol} \mathrm{K}))$. We therefore speculated that the calcium ion might coordinate with several solvents or counter molecules before complexation with ASTX. Once the ASTX/Ca ${ }^{2+}$ complex formed, the boundary molecules on $\mathrm{Ca}^{2+}$ were liberated, and this would account for the positive entropy change.

In summary, the present study demonstrates that transASTX provides binding capacities for two calcium ions and therefore provides a reasonable explanation for the calciuminduced slight increase in absorbance in the UV/vis spectra. The main chelating site is the $\alpha$-hydroxycyclohexenone. Such a calcium-chelating activity of ASTX may provide a rationale for the protective effects of ASTX against calcium-induced lens turbidity ${ }^{9}$ because the presence of ASTX would result in less residual free calcium for calpain activation and would indirectly protect against proteolysis. Moreover, at body temperature $\left(37^{\circ} \mathrm{C}\right)$, a regioselective isomerization of ASTX in the presence of calcium did not change the proportion of 13-cis-ASTX at $48 \mathrm{~h}$. This result may partially explain the reported relative accumulation of the 13-cis isomer over 9-cis and all-trans ASTX in human plasma after supplementation with dietary ASTX. ${ }^{17}$ Nonetheless, the information on how temperature or endogenous metals other than $\mathrm{Ca}^{2+}$ affect the isomerization of ASTX and influence its biological activity in mammals is still limited. We are currently studying the effects of various divalent metals on cis/trans ASTX at various temperatures.

Acknowledgment. The authors thank Dr. Shu-Chuan Jao, Institute of Biological Chemistry, Academia Sinica in Taiwan for helpful discussions on ITC and NMR analyses. This work was supported by the National Science Council, Taiwan (NSC93-2320-B038-053), and the Shin Kong Wu Ho-Su Memorial Hospital, Taiwan (SKH-TMU-96-14).

Note Added after ASAP Publication: In Table 1, sixth column head, the $\mathrm{H}$ should have been a $\mathrm{C}$, this was incorrect in the version published July 13, 2007; it was corrected in the version published July 23, 2007.

Supporting Information Available: UV/vis spectra, Job plot, and HMQC and HMBC spectra of complex formation studies. This material is available free of charge via the Internet at http://pubs.acs.org.

OL0709533

(17) (a) Osterlie, M.; Bjerkeng, B.; Liaaen-Jensen, S. J. Nutr. Biochem 2000, 11, 482-490. (b) Coral-Hinostroza, G. N.; Ytrestoyl, T.; Ruyter, B.; Bjerkeng, B. Comp. Biochem. Physiol., Part C: Toxicol. Pharmacol. 2004, $139,99-110$ 